\title{
The impact of progestin-only contraception on weight change in the first postpartum year
}

\author{
Sara Pentlicky ${ }^{1+*}$, Sarah J Ratcliffe ${ }^{2}$ and Courtney A Schreiber ${ }^{1 *}$ \\ ${ }^{1}$ Department of Obstetrics and Gynecology, Perelman School of Medicine, University of Pennsylvania, 3400 Spruce St., Philadelphia, PA 19104, USA \\ ${ }^{2}$ Department of Biostatistics and Epidemiology, Perelman School of Medicine, University of Pennsylvania, 415 Curie Blvd., Philadelphia, PA, 19104, USA \\ ${ }^{\dagger}$ Current Address: Planned Parenthood of the Great Northwest, 2001 E. Madison St. Seattle, WA 98122, USA
}

\begin{abstract}
Objectives: To investigate the impact of the etonogestrel implant (ENG-IMP) and depot-medroxyprogesterone acetate (DMPA) on weight loss in non-breastfeeding women during the first postpartum year.

Study Design: We designed a modified randomized controlled trial of immediately postpartum, non-breastfeeding women. Sixty six women were randomized to receive either the ENG-IMP or DMPA prior to discharge from the hospital. Thirty four women choosing to leave the hospital without initiating a contraceptive method served as a comparison group. The primary outcome of weight loss, both absolute and percent, was computed, and compared among groups over time during the first postpartum year. Secondary outcomes included contraceptive continuation and satisfaction.

Results: At 3 months postpartum, mean weight loss for ENG-IMP users was 8.1\% (6.5 kg), DMPA users lost 2.2\% (2.0 kg), and the comparison group lost 8.2\% $(6.5 \mathrm{~kg})$. The difference in percent weight change at 3 months between DMPA users and both ENG-IMP users and the comparison group was statistically significant $(\mathrm{p}=0.007, \mathrm{p}=0.02$ respectively). At 6 and 12 months, there were no statistically significant weight loss differences between groups. Randomized women had high rates of satisfaction ("good" or "very good" for 88\% of ENG-IMP users, and 89\% of DMPA users) and continuation (89\% of ENG-IMP and 73\% of DMPA users) at 12 months.

Conclusions: By three months postpartum, women using the ENG-IMP had lost more weight than DMPA users, but the difference was not sustained at 12 months.

Implications: Providers should inform women not breastfeeding in the postpartum period that early administration of DMPA may decrease initial weight loss relative to implant users and those getting DMPA later, though weight loss at 12 months postpartum may be the same among all groups. This study highlights the relatively small weight losses in the postpartum period among all groups and indicates the need for education and programs to encourage postpartum weight loss in this population.
\end{abstract}

Abstract: 309 words

\section{Introduction}

Obesity and unintended pregnancy are major public health problems in the United States. According to the Centers for Disease Control and Prevention (CDC), approximately two-thirds of US women are overweight or obese and approximately 3 million women experience an unintended pregnancy annually $[1,2]$. The postpartum period presents a critical time to address both weight management and healthy pregnancy spacing. Data suggest that excessive pregnancy weight gain and postpartum weight retention are risk factors for future obesity [3-7]. In one study, women who had not returned to their prepregnancy weight by six months postpartum were $6 \mathrm{~kg}$ heavier 8 years later than women who had quickly reached their pre-pregnancy weight $(\mathrm{p}=.01)$ [6,7]. Furthermore, rapid repeat pregnancy (RRP) has been associated with weight gain over time, in addition to many other poor maternal-child health outcomes [8-10].

Progestin-only contraceptive methods are favored postpartum because they do not increase the risk of venous thromboembolism (VTE) or interfere with breastfeeding [11-14]. Depot medroxyprogesterone acetate (DMPA), the injectable contraceptive available in the US, is heavily utilized postpartum, but is associated with weight gain in some populations outside of pregnancy $[15,16]$. Post-marketing data on the etonogestrel implant (ENG-IMP) provide some evidence that it is associated with weight gain [17]. The primary objective of this study was to determine if women who use these 2 highly-effective progestinonly contraceptives immediately postpartum lose pregnancy weight differently than each other, and/or differently than women who leave the hospital without initiating such methods.

\section{Materials and methods}

To compare percent weight loss among the 3 study groups, we conducted a modified randomized controlled trial at the Hospital of the University of Pennsylvania (HUP) from 2011-2012. Healthy women aged 18 to 45 , postpartum of a neonate greater than or equal to 37 weeks gestation, proficient in English and planning to delay another pregnancy for at least six months were eligible. Women were

Correspondence to: Courtney A. Schreiber, MD, MPH, Penn Family Planning and Pregnancy Loss Center, 1000 Courtyard, 3400 Spruce St, Philadelphia, PA 19104, USA, Tel: 215-615-6531; Fax: 215-615-5319; E-mail: schreibe@upenn.edu

Key words: contraception, postpartum, progestin-only, weight loss

Received: March 15, 2018; Accepted: March 27, 2018; Published: April 02, 2018 
excluded if they were breastfeeding, unable to attend follow up visits, or planned to use weight loss medications during the study. Eligible and willing postpartum women were randomly assigned to receive either ENG-IMP or DMPA before hospital discharge. Women who declined to start a contraceptive method before leaving the hospital, but were otherwise interested and eligible, were offered the opportunity to participate in a comparison group. We designed the study to include a comparison group of women not immediately starting a hormonal contraceptive method and allowed them to self-select this comparison arm because we did not consider it ethical to randomize women to no postpartum contraceptive plan upon discharge. This study design was successfully utilized previously [18]. Lastly, as a standard part of care, ENG-IMP and DMPA are offered prior to discharge in the hospital or at a postpartum follow-up visit with no cost to the patient, thus access to these contraceptives was not an incentive to participate. We reimbursed women for their time and travel during the study. The Institutional Review Board of the University of Pennsylvania approved our study.

Because the expected amount of postpartum weight loss in nonbreastfeeding women is not documented in the literature [19], we chose a sample size that would enable us to detect a one standard deviation difference in weight loss between the 3 groups at the primary 6 month endpoint. This required a minimum of 22 women in each group. With a predicted $34 \%$ loss to follow-up [20], our calculated sample size was 100 with 34 women in the comparison group arm, and 33 in each of the ENG-IMP and DMPA arms.

The study flow is illustrated in Figure 1. Block randomization was utilized. Participants could discontinue, switch, or initiate any method at will during the study period. All data for each group, including both primary and secondary outcomes, were analyzed as assigned, except satisfaction, which was analyzed with only women still using their assigned method. Any woman who became pregnant during the study period was discontinued from the study. Missed interim visits were counted as missing data.

All subjects were weighed at enrollment before being discharged from the hospital. After enrollment, subjects returned at 3, 6 and 12 months postpartum for a weight measurement, a urine pregnancy test and a questionnaire. Weight was recorded for each woman at each visit, including enrollment, in her own clothing without shoes or outerwear,

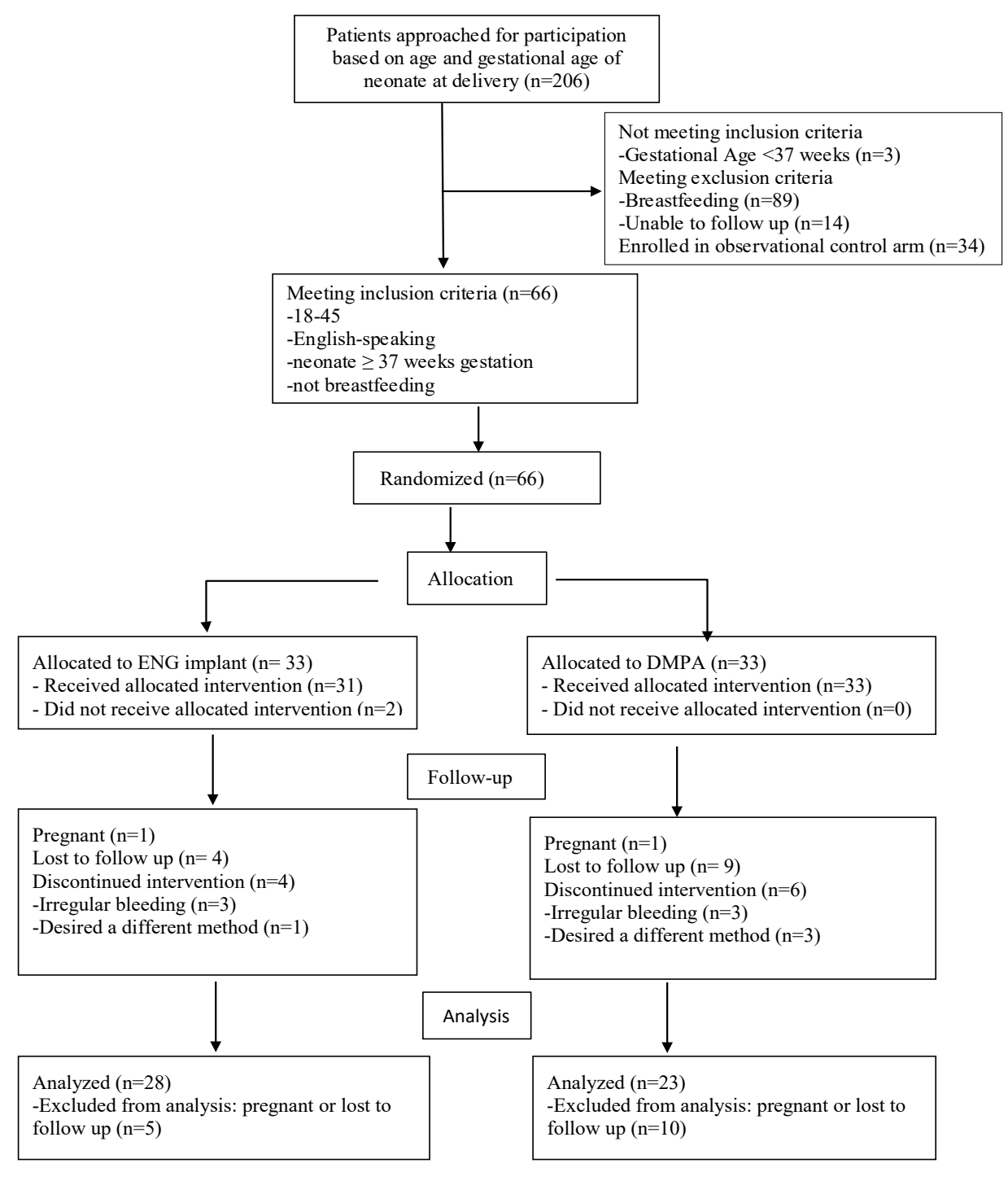

Figure 1. CONSORT flow diagram. 
using one calibrated scale (A\&P Medical, Carrollton, TX). Trained research staff completed questionnaires at enrollment and each followup visit.

Weight in the postpartum period is subject to many influences, including fluid shifts immediately postpartum, breastfeeding, exercise and diet. Ranges of body mass index (BMI) that denote normal or excess weight have not been validated during or after pregnancy; however BMI can provide a way to conceptualize and frame weight-forheight. We chose to evaluate BMI at enrollment; however we ultimately evaluated percent weight loss as our primary outcome measure.

Participants completed previously validated surveys that included questions relating to physical activity and diet at each of the 3 follow-up visits. Physical activity questions were obtained from the World Health Organization (WHO) Global Physical Activity Questionnaire (GPAQ). The GPAQ includes a validated method of interpreting these questions to assign levels of physical activity [21]. This tool accumulates all daily activity, recreational as well as physical activity acquired through work or travel to and from work. The tool provides a mechanism to estimate mean activity in each group. We used the "Weight and Lifestyle Inventory" (WALI), a previously validated tool to obtain diet history [22]. Specifically, we implemented the WALI 24-hour recall diary at each follow-up interview. Participants chose 1 or 2 days in the last week that represent their typical diet including all foods and beverages consumed. While subject to recall bias, it was a frequently used tool that required only short term recall and allowed for at least 2 different days of food consumption in case there were outliers in that week. Calorie counts were totaled for each day and averaged, giving an approximate daily caloric intake for each participant. This was repeated at all follow up appointments. Overall net caloric intake and expenditure were calculated for each study time point. Participants also reported satisfaction with each contraceptive method (assessed with Likert 5-scale questions).

We entered responses directly into Research Electronic Data Capture, or REDCap ${ }^{\oplus}$ [23]. Descriptive statistics summarized the demographic data overall, and by study arm. We compared demographic characteristics between study arms using ANOVA / Kruskal-Wallis test or Fisher's exact tests. The primary outcome of percentage weight change from baseline to 6 months was calculated for each woman. Oneway ANOVA, with Tukey adjustment for multiple comparisons, was used to test for differences in weight change. Generalized estimating equations (GEEs) were used to test for differences among all 3 groups over all the study periods, with Holm's adjustments for multiple comparisons [24-26]. The GEE analysis of weight loss tested for a group by time interaction, adjusting for other potential covariates/confounders (race, calories consumed and physical activity) that could be associated with weight loss. Time was modeled as a non-linear function. All analyses were based on intention-to-treat principles.

\section{Results \\ Weight loss}

One hundred women enrolled in our prospective trial of postpartum weight loss: 33 randomized to the ENG-IMP, 33 randomized to DMPA and 34 in the comparison group (Figure 1). All groups were similar except that women in the randomized cohorts were, on average, three years younger than the comparison group $(\mathrm{p}=0.002)$ and more likely to be black $(\mathrm{p}<.001)$ (Table 1$)$. At the 3 and 6 month follow-up visits there were 79 and 73 women remaining in the study, respectively. This demonstrated a $27 \%$ loss to follow-up rate at the end of 6 months. There were no statistically significant differences in baseline characteristics between those who were lost to follow-up and those with complete data, at 3 months, or at 6 months (all p>0.1). At 12 months, 67 of the initial 100 women remained in the study, 28 in the ENG-IMP group, 23 in the DMPA group, and 16 in the comparison group. This represents an overall $33 \%$ percent loss to follow-up, which was significantly higher $(\mathrm{p}=0.004)$ among those in the comparison group.

Overall mean percent weight lost at 3 months postpartum was $6.3 \%$. The mean loss for the comparison group and ENG-IMP group was $8.2 \%(6.5 \mathrm{~kg})$ and $8.1 \%(6.5 \mathrm{~kg})$ respectively, while the DMPA group lost $2.2 \%(2.0 \mathrm{~kg})$ (Table 2). In adjusted models, differences at 3 months were statistically significant (ENG-IMP vs. DMPA $p=0.007$, DMPA vs. comparison group $\mathrm{p}=0.02$ ). At 6 months postpartum, the DMPA group showed a $3.7 \%$ decrease in weight from postpartum. However, at this time point, mean weight loss for the ENG-IMP group and the comparison group showed that both groups, on average, gained weight during the 3-6 month period. At 1 year, DMPA users had lost a total of $4.2 \%$ of their total body weight from their initial weight postpartum. The ENG-IMP group lost $6.0 \%$ and the comparison group $8.2 \%$. None of these group differences after 3 months postpartum were statistically significant.

While each group lost weight over time, not every individual lost weight. The DMPA group had a steady decrease in percent weight lost across the 12 months, while the ENG-IMP and the comparison group mirrored each other and had substantial weight loss at the 3-month time point but then plateaued (Table 2). These group differences in weight loss trajectories, assessed by GEE, had a significance of $\mathrm{p}=0.64$.

\section{Caloric intake and expenditure}

There were no statistically significant differences in caloric intake among any group at any time point. Median physical activity was constant over time in the comparison group but increased in ENGIMP users (4080 to 10080 weekly metabolic equivalents, $\mathrm{p}=0.048$ ) and in DMPA users (6300 to 10080 weekly metabolic equivalents, $\mathrm{p}<.005$ ) over 12 months. Further, DMPA users exerted approximately double the amount of physical activity as the comparison group at 12 months ( 10080 vs. 6380 weekly metabolic equivalents, $\mathrm{p}=0.006$ ).

\section{Contraceptive method continuation}

At 3 months, all women randomized to receive the ENG-IMP (29 of 29) or DMPA (24 of 24), who remained in the study, continued their assigned method. At 6 months, 93\% (25 out of 27) of initial ENG-IMP recipients retained their implant and 76\% (17 out of 25) participants randomized to DMPA were still using DMPA. At 12 months 89\% (24 out of 27) of women randomized to the ENG-IMP and 74\% (17 out of 23) of women randomized to DMPA reported that they were using their assigned method (Fisher exact test comparison $\mathrm{P}=0.27$ ).

\section{Satisfaction with contraceptive method}

At 12 months, 15 of the 17 women (89\%) assigned to and still using DMPA reported a satisfaction level of "good" or "very good". Of the 24 women assigned to and still using the ENG-IMP at 12 months, 21 (88\%) reported the same level of satisfaction. Eighty-one percent of women in the comparison group at 12 months $(n=16)$ reported their satisfaction level as "good" or "very good". Women randomized to ENG-IMP were more likely than DMPA users or comparison group to recommend their method to a friend at 3, 6 and 12 months, $\mathrm{p}<.001, \mathrm{p}=.03$ and $\mathrm{p}=.005$, respectively. At 12 months, $92 \%$ of those 24 women in the ENG-IMP group stated they were "very likely" to recommend the implant to a friend. The remaining 2 women stated they were "somewhat likely". 
Table 1. Baseline demographic characteristics.

\begin{tabular}{|c|c|c|c|c|c|}
\hline Characteristic & & $\begin{array}{c}\text { Comparison group } \\
\mathrm{N}=34\end{array}$ & $\begin{array}{c}\text { ENG implant } \\
\mathrm{N}=33\end{array}$ & $\begin{array}{c}\text { DMPA } \\
\mathrm{N}=33\end{array}$ & $\begin{array}{c}\text { All group } \\
\text { p- value }\end{array}$ \\
\hline Age (yr) & & $26.6(5.4)$ & $22.4(3.8)$ & $23.1(4.7)$ & .002 \\
\hline \multicolumn{2}{|c|}{ Baseline Weight (kg) } & $80.3(17.6)$ & $81.9(17.9)$ & $81.1(24.8)$ & .89 \\
\hline \multicolumn{2}{|c|}{ BMI (median, range) } & $28.7(21.6,43.7)$ & $28.7(20.7,53.7)$ & $29.7(20.6,58.2)$ & .68 \\
\hline \multirow[t]{3}{*}{ Education } & $<$ High School & $7(21)$ & $7(21)$ & $11(33)$ & \multirow{3}{*}{.48} \\
\hline & High School/GED & $15(44)$ & $18(55)$ & $16(49)$ & \\
\hline & $>$ High School & $12(35)$ & $8(24)$ & $6(18)$ & \\
\hline \multirow[t]{3}{*}{ Race } & Black & $23(68)$ & $31(94)$ & $29(88)$ & \multirow{3}{*}{$<.001$} \\
\hline & White & $10(29)$ & $0(0)$ & $1(3)$ & \\
\hline & Other & $1(3)$ & $2(6)$ & $3(9)$ & \\
\hline \multirow[t]{2}{*}{ Ethnicity } & Hispanic & $2(6)$ & $0(0)$ & $3(9)$ & \multirow{2}{*}{.28} \\
\hline & Not Hispanic & $32(94)$ & $33(100)$ & $30(91)$ & \\
\hline \multirow[t]{5}{*}{ Income } & $<\$ 10,000$ & $7(21)$ & $6(18)$ & $13(39)$ & \multirow{5}{*}{.39} \\
\hline & $\$ 10,001-\$ 20,000$ & $7(21)$ & $6(18)$ & $8(24)$ & \\
\hline & $\$ 20,001-\$ 40,000$ & $5(15)$ & $6(18)$ & $3(9)$ & \\
\hline & $>\$ 40,001$ & $7(21)$ & $5(15)$ & $2(6)$ & \\
\hline & unsure & $8(24)$ & $10(30)$ & $7(21)$ & \\
\hline \multirow[t]{3}{*}{ Parity } & 1 & $11(32)$ & $10(30)$ & 13(39) & \multirow{3}{*}{.36} \\
\hline & 2 & $11(32)$ & $13(39)$ & $5(15)$ & \\
\hline & $>=3$ & $12(35)$ & $10(30)$ & $15(46)$ & \\
\hline \multirow[t]{3}{*}{ Pregnancy Prevention } & Extremely/ Very Important & $28(82)$ & $29(88)$ & $29(88)$ & \multirow{3}{*}{.95} \\
\hline & $\begin{array}{l}\text { Important/Somewhat } \\
\text { Important }\end{array}$ & $5(15)$ & $3(9)$ & $4(12)$ & \\
\hline & Not Important & $1(3)$ & $1(3)$ & $0(0)$ & \\
\hline \# of years until & $<=5$ years & $10(29)$ & $7(21)$ & $11(33)$ & \multirow{3}{*}{.17} \\
\hline next baby & 6 or more years & $10(29)$ & $17(52)$ & $7(21)$ & \\
\hline desired & Never & $14(41)$ & $9(27)$ & $15(46)$ & \\
\hline
\end{tabular}

$\mathrm{N}(\%)$ or mean $( \pm \mathrm{SD})$ if not stated. All Group comparisons used ANOVA / Kruskal-Wallis test or Fisher's exact tests as appropriate.

Table 2. Postpartum weight change among the three study groups.

\begin{tabular}{|c|c|c|c|c|c|c|}
\hline & \multicolumn{2}{|c|}{3 months } & \multicolumn{2}{|c|}{6 months } & \multicolumn{2}{|c|}{12 months } \\
\hline & $\mathrm{N}$ & $\begin{array}{c}\text { Weight change } \\
\%\end{array}$ & $\mathrm{~N}$ & $\begin{array}{c}\text { Weight change } \\
\%\end{array}$ & $\mathrm{~N}$ & $\begin{array}{c}\text { Weight change } \\
\%\end{array}$ \\
\hline DMPA & 24 & $-2.2 \pm 3.8$ & 25 & $-3.7 \pm 3.6$ & 23 & $-4.2 \pm 3.9$ \\
\hline ENG-IMP & 29 & $-8.1 \pm 2.0$ & 27 & $-7.0 \pm 2.7$ & 28 & $-6.0 \pm 3.9$ \\
\hline Comparison group & 26 & $-8.2 \pm 3.6$ & 21 & $-7.2 \pm 4.9$ & 16 & $-8.2 \pm 5.9$ \\
\hline
\end{tabular}

Weight change (mean $\pm \mathrm{SD}$ ) is \% of postpartum weight at discharge. GEE analysis adjusted for age, race, calories consumed and physical activity. At 3 months, mean percent weight change was lower for the DMPA users compared to the ENG-Implant users ( $\left.\mathrm{p}_{\text {holms }}=0.007\right)$ and controls $\left(\mathrm{p}_{\text {holms }}=0.02\right)$. There were no significant differences between groups at 6 or 12 months.

\section{Pregnancy}

There were six pregnancies during the first postpartum year, four occurred in the comparison group. One woman in the ENG-IMP arm and 1woman in the DMPA arm became pregnant and both had discontinued the method before the pregnancy was conceived. All women who became pregnant during the study period reported that the pregnancies were unintended and/or mistimed. Upon learning about this pregnancy women reported feeling: overwhelmed $(5 / 6)$, scared (3/6), sad (2/6), angry (2/6) and regret (2/6). One woman reported feeling nothing, and no woman reported feeling happy about her new pregnancy.

\section{Discussion}

Progestin-only methods are safe for use in the immediate postpartum period and are available in highly-effective, long-acting delivery systems that can improve child spacing [27]. We designed this study to assess the impact of these contraceptive methods on postpartum weight loss. We found that women using the implant lost weight more quickly than DMPA users. Women who were randomized to immediate postpartum DMPA retained on average $4.0 \%$ more weight than the comparison group and $1.8 \%$ more than those randomized to the ENG-IMP at 1 year post-delivery. While this difference was not statistically significant in our cohort of 100 women, it deserves consideration given that the Centers for Disease Control and Prevention (CDC) and the National Institutes of Health (NIH) suggest that outside of pregnancy and postpartum periods, a 5-10\% decrease in body weight can provide major health benefits [28].

Our study has limitations. First, given the highly variable nature of breastfeeding frequency, and its complex relationship to caloric intake and expenditure, we chose to exclude breastfeeding women from this initial study. Thus, our findings may not be generalizable to the breastfeeding population. Future studies that include breastfeeding women are needed to better understand the impact of progestin-only contraceptives on weight loss trajectories in all postpartum women. Second, we collected all primary outcome data prospectively at the time of delivery in order to avoid recall bias, but the associated limitation is that we did not have pre-pregnancy weight for any participant. While we therefore could not compute the number of women who returned to their pre-pregnancy weight by the $12^{\text {th }}$ postpartum month, we observed that implant users and the comparison group experienced a weight loss plateau after 3 months postpartum. Our comparison group, a heterogeneous group using a variety of contraceptive methods, lost 
the most weight on average, but the mean percent weight lost in this group was roughly $7 \%$, which for our population was an average of $5.4 \mathrm{~kg}$ (12 pounds) at one year postpartum. It is unlikely that a 12 pound weight loss at 12 months postpartum represents a return to pre-pregnancy weight, given the current CDC guidelines recommend a 25-35 pound weight gain. Furthermore, many individuals plateaued or gained weight in the second half of the study period.

Strengths of our study include the randomized design, which allowed us to minimize weaknesses such as confounding by indication. Our satisfaction data show that not only are women willing to be randomized to a contraceptive method; but that, once randomized, women who were assigned their method were happier with their method than women in the comparison group who were using a method (or no method) that they chose. We also observed high continuation rates of both the ENG-IMP and DMPA, suggesting method acceptability, given that these women were contacted frequently, and could have discontinued at any time. Lastly, this study contributes 12-month follow-up data, which provides a longitudinal picture of the first postpartum year, including point estimates for postpartum weight loss throughout that year.

Side effects attributed to contraceptives, including weight changes, are common reasons for discontinuation. This preliminary study demonstrates that when comparing the ENG-IMP to DMPA, postpartum weight loss appears to be initially faster with the ENGIMP, but no different at 12 months postpartum. Both The American Congress of Obstetricians and Gynecologists [29] and the American Academy of Pediatrics [30] recommend long acting reversible methods as first line contraceptives. Women who desire a user-friendly method that allows for optimal child-spacing can be reassured that the ENGIMP does not impede postpartum weight loss.

\section{Acknowledgements}

We would like to thank the anonymous donor for supporting this research. We would also like to thank Kurt Barnhart MD, MSCE for his guidance through all stages of this research. We express appreciation to those who were crucial in conducting this project: Veronica Chavez, Monica Ionescu, Carrie Miller, Emilia Flores Rabinowitz. The authors declare that they have no competing interests'.

\section{Clinical Trials.gov identifier}

NCT02144259, The impact of contraception on postpartum weight loss.

\section{References}

1. Flegal KM, Carroll MD, Kit BK, Ogden CL (2012) Prevalence of obesity and trends in the distribution of body mass index among US adults, 1999-2010. JAMA 307: 491497. [Crossref]

2. Finer LB, Zolna MR (2014) Shifts in intended and unintended pregnancies in the United States, 2001-2008. Am J Public Health 104 Suppl 1: S43-S48. [Crossref]

3. Mamun AA, Kinarivala M, O'Callaghan MJ, Williams GM, Najman JM, et al. (2010) Associations of excess weight gain during pregnancy with long-term maternal overweight and obesity: evidence from 21 y postpartum follow-up. Am J Clin Nutr 91: 1336-1341. [Crossref]

4. Gore SA, Brown DM, West DS (2003) The role of postpartum weight retention in obesity among women: a review of the evidence. Ann Behav Med 26: 149-159. [Crossref]

5. Gunderson EP, Abrams B (2000) Epidemiology of gestational weight gain and body weight changes after pregnancy. Epidemiol Rev 22: 261-274. [Crossref]

6. Rooney BL, Schauberger CW (2002) Excess pregnancy weight gain and long-term obesity: one decade later. Obstet Gynecol 100: 245-252. [Crossref]

7. Schauberger CW, Rooney BL, Brimer LM (1992) Factors that influence weight loss in the puerperium. Obstet Gynecol 79: 424-429. [Crossref]
8. Shachar BZ, Lyell DJ (2012) Interpregnancy interval and obstetrical complications. Obs Gynecol Surv 67: 584-596. [Crossref]

9. Conde-Agudelo A, Rosas-Bermúdez A, Kafury-Goeta AC (2006) Birth spacing and risk of adverse perinatal outcomes: a meta-analysis. JAMA 295: 1809-1823. [Crossref]

10. Conde-Agudelo A, Belizán JM (2000) Maternal morbidity and mortality associated with interpregnancy interval: cross sectional study. BMJ 321: 1255-1259.

11. Brito MB, Ferriani RA, Quintana SM, Yazlle ME, Silva de Sa MF, et al. (2009) Safety of the etonogestrel-releasing implant during the immediate postpartum period: a pilot study. Contraception 80: 519-526. [Crossref]

12. Gurtcheff SE, Turok DK, Stoddard G, Murphy PA, Gibson M, et al. (2011) Lactogenesis after early postpartum use of the contraceptive implant: a randomized controlled trial. Obstet Gynecol 117: 1114-1121. [Crossref]

13. Jimenez J, Ochoa M, Soler MP, Portales P (1984) Long-term follow-up of children breast-fed by mothers receiving depot-medroxyprogesterone acetate. Contraception 30: 523-533. [Crossref]

14. Pardthaisong T, Yenchit C, Gray R (1992) The long-term growth and development of children exposed to Depo-Provera during pregnancy or lactation. Contraception 45: 313-324. [Crossref]

15. Bonny AE, Secic M, Cromer B (2011) Early weight gain related to later weight gain in adolescents on depot medroxyprogesterone acetate. Obstet Gynecol 117: 793-797. [Crossref]

16. Espey E, Steinhart J, Ogburn T, Qualls C (2000) Depo-provera associated with weight gain in Navajo women. Contraception 62: 55-58. [Crossref]

17. Nault AM, Peipert JF, Zhao Q, Madden T, Secura GM (2013) Validity of perceived weight gain in women using long-acting reversible contraception and depot medroxyprogesterone acetate. Am J Obstet Gynecol: 208. [Crossref]

18. Design of the Women's Health Initiative clinical trial and observational study (1998) The Women's Health Initiative Study Group. Control Clin Trials 19: 61-109.

19. Institute of Medicine (U.S.) Committee on Nutritional Status During Pregnancy and Lactation. Subcommittee for a Clinical Application (1992) Nutrition during pregnancy and lactation: an implementation guide. Natl Acad Press.

20. Schreiber CA, Ratcliffe SJ, Barnhart KT (2010) A randomized controlled trial of the effect of advanced supply of emergency contraception in postpartum teens: a feasibility study. Contraception 81: 435-440. [Crossref]

21. Bull FC, Maslin TS, Armstrong T (2009) Global physical activity questionnaire (GPAQ): nine country reliability and validity study. J Phys Act Heal 6: 790-804. [Crossref]

22. Wadden TA, Foster GD (2006) Weight and Lifestyle Inventory (WALI). Surg Obes Relat Dis 2: 180-199.

23. Data capture (REDCap) (2009) A metadata-driven methodology and workflow process for providing translational research informatics support. J Biomed Inf 42: 377-381.

24. Liang KY, Zeger SL (1986) Longitudinal data analysis using generalized linear models. Biometrika 73: 13-22.

25. Zeger S, Liang KY (1986) Longitudinal data analysis for discrete and continuous outcomes. Biometrics 42: 121-130.

26. Holm S (1979) A Simple Sequentially Rejective Multiple Test Procedure. Scand J Stat 6: $65-70$.

27. Peipert JF, Madden T, Allsworth JE, Secura GM (2012) Preventing Unintended Pregnancies by Providing No-Cost Contraception. Obstet Gynecol 120: 1291-1297. [Crossref]

28. Blackburn G (1995) Effect of degree of weight loss on health benefits. Obes Res 3 Suppl 2: 211s-216s. [Crossref]

29. American College of Obstetrics and Gynecology (ACOG) (2009) ACOG Committee Opinion no. 450: Increasing use of contraceptive implants and intrauterine devices to reduce unintended pregnancy. Obstet Gynecol 114: 1434-1438. [Crossref]

30. Statement P, COMMITTEE ON ADOLESCENCE (2014) Contraception for Adolescents | Pediatric Annals. Pediatrics 134: e1244-56.

Copyright: (C2018 Pentlicky S. This is an open-access article distributed under the terms of the Creative Commons Attribution License, which permits unrestricted use, distribution, and reproduction in any medium, provided the original author and source are credited. 